\title{
Correction to: The association between the inflammatory potential of diet and risk of developing, and survival following, a diagnosis of ovarian cancer
}

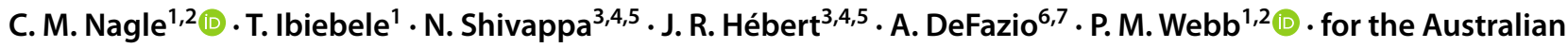 \\ Ovarian Cancer Study
}

Published online: 28 September 2018

(c) Springer-Verlag GmbH Germany, part of Springer Nature 2018

\section{Correction to: European Journal of Nutrition https://doi.org/10.1007/s00394-018-1779-x}

In the original publication of this article on page 6, paragraph "Discussion", line 4, 'In a U.S. population-based case-control study ( $n=493$ cases) Peres et al., reported a non-significant association between DII score and risk of developing ovarian cancer of similar magnitude (OR DII score $_{\mathrm{Q} 4 \text { vs. Q1 }} 1.35,95 \%$ CI 0.93-1.97) [20]'. It should read as 'In a U.S. population-based case-control study $(n=493$ cases) Peres et al., reported a significant association between DII score and risk of developing ovarian cancer (OR DII score $_{\mathrm{Q} 4 \text { vs. Q1 }} 1.72,95 \%$ CI 1.18-2.51) [20]'. The original article has been corrected.

The original article can be found online at https://doi.org/10.1007/ s00394-018-1779-x.

C. M. Nagle

christina.nagle@qimrberghofer.edu.au

1 Gynaecological Cancers Group, QIMR Berghofer Medical Research Institute, Locked Bag 2000, Royal Brisbane Hospital, Herston, QLD 4029, Australia

2 Faculty of Medicine, School of Public Health, The University of Queensland, Brisbane, Australia

3 Cancer Prevention and Control Program, University of South Carolina, 915 Greene Street, Suite 241-2, Columbia, SC 29208, USA

4 Department of Epidemiology and Biostatistics, Arnold School of Public Health, University of South Carolina, 915 Greene Street, Suite 400, Columbia, SC 29208, USA

5 Connecting Health Innovations LLC (CHI), 1417 Gregg Street, Columbia, SC 29201, USA

6 Centre for Cancer Research, The Westmead Institute for Medical Research, The University of Sydney, Sydney, Australia

7 Department of Gynaecological Oncology, Westmead Hospital, Sydney, Australia 